\title{
Papà non è Babbo Natale
}

\author{
Susanne Schulze-Boysen \\ Escuela de Lenguas Modernas \\ Universidad de Costa Rica
}

\begin{abstract}
Riassunto
Prendendo spunto dalla lettura di due romanzi abbastanza conosciuti e peraltro trasferiti sul grande schermo, si considera il rapporto "padre-figlio" sotto l'aspetto del sacrificio del figlio da parte del padre. Cosa spinge un padre a sacrificare un figlio? Si analizzano esempi tanto nella letteratura quanto nella vita reale.
\end{abstract}

Parole chiave: sacrificio, padre, figlio, incomprensione, comunicazione

\begin{abstract}
Based on two fairly popular novels which were also produced on screen, this essay analyzes the relationship between father and son with regards to the father's sacrificing his son. What makes a father sacrifice his son? The article analyzes some literary as well as real life examples.
\end{abstract}

Key words: sacrifice, father, son, misunderstanding, communication

\section{Introduzione}

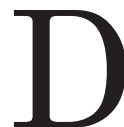

opo aver letto Io non ho paura di Niccoló Ammaniti (1966), con il suo finale impattante, si è presentato il paragone con The Boy In The Striped Pyjamas di John Boyne (1971). I due libri hanno in comune il sacrificio di un figlio. Più avanti nell'articolo si paragonano i due film nella letteratura e le loro tematiche nella vita reale. I due film sono stati portati sul grande schermo, con i relativi adattamenti interpretativi. Il film italiano non trasmette né la gravità della situazione né una possibile finale tragica. Il film britannico-irlandese del ragazzo con il pigiama a righe, prodotto nel 2008, è stato un successo mondiale, ma non ha comunque trasmesso la complessità dei pensieri del bambino; inoltre il rapporto della madre con il giovane tenente, appena accennato nel libro, nel film si manifesta più evidente. 


\section{lo non ho paura}

È la storia di un ragazzo di nove anni, Michele, che vive in un paesino sperduto del Sud Italia durante l'estate del 1978. In questo paesino di quattro case e una villa signorile vivono cinque famiglie e i bambini costituiscono quasi obbligatoriamente un gruppo di giochi unito, dove il più grande, chiamato il Teschio, di dodici anni, è un leader più o meno sadico. Le quattro famiglie contadine vivono in una povertà relativa, in case piccole ma funzionali, dove il cibo non manca. L'acqua viene raccolta dalla fontana in piazza, ma nelle case si trova la corrente elettrica e la televisione; l'estate è calda, estenuante e torrida e di giorno soltanto i bambini escono all'aria aperta.

Il padre di Michele è camionista e trasporta grano al nord. Un altro fra i padri del vicinato è barbiere e contadino, mentre fra le madri una ha un piccolo spaccio di prime necessità, e il capo famiglia della villa è un avvocato che lavora a Roma. La madre di Michele non si riposa mai: accudisce alla famiglia e alla casina di due stanze da letto, una sala ed una cucina.

Michele racconta la storia in prima persona e si dimostra fin dall'inizio debole e forte nello stesso tempo. In una gara persa per colpa della sorellina gli viene data una penitenza dal Teschio: deve attraversare una casa diroccata, scoperta per caso dai ragazzi. Nonostante la grande paura riesce a inoltrarsi nella casa e scopre un buco coperto da una tettoia di plastica verde, così sposta la lastra e appare una gamba bianca di bambino. Si spaventa moltissimo e pensa che sia di un bambino morto. Però la curiosità ha la meglio su di lui, così ritorna da solo e scendendo nel buco scopre che il bambino è vivo $\mathrm{e}$ legato alla caviglia con un catenaccio. Gli viene dato da mangiare e da bere in una ciotola di casa sua. Rimuginando immagina che sia un suo fratello gemello nato con qualche problema, perciò rifiutato dalla madre e nascosto dal padre nel buco. Il bambino è molto debole, sporco e malandato e parla di cose che Michele non riesce a capire, così riflette sulla risurrezione di Lazzaro e sul fatto che solamente Gesù può ridare la vita.

La sera gli uomini sono riuniti nella sala e guardano il telegiornale, dove c'è una donna che implora i rapinatori di restituirgli suo figlio, di non fargli alcun male e non tagliargli un orecchio. Il padre di Michele dice che gliele taglierà entrambe e Michele intuisce dalla foto che si tratta del bambino scoperto da lui nel buco.

Il giorno dopo visita il bambino per dargli da mangiare e bere e per dirgli che sua madre lo sta cercando e gli vuole bene. Filippo, così si chiama, non ci crede: per spiegarsi quello che gli è successo pensa che lui e tutta la sua famiglia siano morti e messi in dei buchi come quello in cui si trova lui. Da parte sua Michele comincia a cambiare la percezione del padre come capo del paese; la sera prima un ospite di Roma aveva insultato il padre e lui non si era difeso. Lo immagina inoltre come tagliatore di orecchie di bambini.

Quando fa visita a Salvatore, il suo miglior amico, svela il suo segreto in cambio di figurine di una squadra di calcio. Poi va da Filippo per portargli un dolce e delle caramelle. Scende e gli promette di tornare di nuovo; viene sorpreso da Felice, il fratello grande del Teschio, altra figura nefasta. Questi lo malmena e lo mette nella sua auto 
dove c'è Salvatore, che gli ha svelato il segreto in cambio del piacere di poter guidare un pochino la sua macchina.

La madre di Michele, all'arrivo di suo figlio in quello stato, se la prende con Felice e gli sferra un calcio nelle parti basse. Quando Felice si riprende, comincia a lottare con la madre, una bella donna, e nel trambusto le scivola il vestito lasciandola quasi nuda. Arriva il padre con il suo amico, vede la scena e prende a botte Felice. Michele passa la notte confortato da sua madre. Il giorno dopo, il padre gli fa giurare che non andrà più a vedere il bambino nascosto e gli promette che presto andranno al mare e che si trasferiranno al nord.

Giocando di nuovo, Michele fa pace con Salvatore; in cambio Salvatore gli confida di aver sentito che il bambino non era più nel buco, ma lo avevano messo nella gravina di un fattore.

La stessa sera Michele sente dei litigi in salotto; riesce a capire che uno dei quattro uomini presenti sparerà al ragazzo perché il sequestro non ha avuto i risultati sperati.

$\mathrm{Al}$ mattino vince nuovamente paure e timori, e va per salvare Filippo. Con molta abilità riesce ad aprire il nascondiglio e convince Filippo a scappare, ma per una lesione alla caviglia non riesce a uscire lui stesso. In quel momento appare suo padre con una pistola in mano. Il padre gli spara.

Più per fortuna del padre che per Michele, questi non muore per mancato colpo e potrà raccontare la storia già da adulto.

Il padre potente e protettore si trasforma in padre aggressore e omicida, vittima della figura di sé, che non vuole screditarsi davanti ai suoi complici. Quando aveva suggerito ai complici di tralasciare la faccenda del rapimento è stato deriso. Si tratta della frantumazione e del crollo della figura paterna.

Qual è stato il motivo per cui il padre ha partecipato al rapimento di un bambino? La famiglia viveva tranquilla, povera sì, ma non in miseria. Il fatto di viaggiare al nord e di aver conosciuto un altro tipo di vita, più comoda e agiata da sembrare televisiva, possono averlo spinto a unirsi ad un bandito e a trascinare con sé i compaesani di Acqua Traverse.

All'inizio il padre, di ritorno dal nord, aveva riempito la casa di gioia, portando regali e giocando con i figli. Quando Michele aveva scoperto il bambino nel buco il primo impulso era stato di raccontare tutto al padre, poi si era frenato e aveva deciso di mantenere il segreto tutto per sé.

\section{The Boy in the Striped Pyjamas (II ragazzo col pigiama a righe)}

Bruno, bambino di nove anni, non capisce perché la famiglia debba traslocare dalla bella casa a Berlino. Sente che il Fury (non capisce la parola Führer) ha grandi progetti per suo padre, e assieme alla sorella grande e alla mamma lo seguono in un treno diretto all'est. Arrivato in una casa sperduta si ribella perché è brutta e non c'è nessun posto dove andare né amici con cui giocare, ma i genitori non tollerano il suo rifiuto e così si ambienta. Dalla sua finestra guarda oltre il giardino e vede un recinto altissimo e lunghissimo con filo spinato, e più oltre vede baracche e molta gente, da bambini a vecchi, tutti tristi e vestiti in pigiama a righe grigie. Capisce che il posto dove sono si chiama Out-With. Sua sorella interpreta infatti Auschwitz come out, fuori da un 
luogo dove una persona non ha fatto bene il suo lavoro.

Il padre è una persona seria e elegante nella sua uniforme perfettamente stirata. Lavora in un ufficio dove per Bruno vale sempre il fermo divieto di entrare, per nessuna ragione. Vanno e vengono molti soldati. In un momento di solitudine Bruno bussa per entrare e suo padre non si arrabbia. Bruno gli confida il suo sgomento e il desiderio di tornare a casa loro a Berlino, ma il padre gli risponde che la casa è dove si trova la famiglia e che per il momento la sua casa è lì. Bruno allora gli chiede se ha combinato qualche gran guaio per essere stato mandato in quel posto per punizione, ma il padre gli risponde che lui non può capire e gli ordina di tornare in camera sua. Bruno domanda nuovamente riguardo alle persone che vede dalla finestra, ma il padre risponde che quella che lui vede non è gente nel vero senso della parola e che non hanno niente a che fare con lui.

Cercando di farsi alleata la domestica, Bruno definisce il padre uno stupido, ma questa protesta dicendo che con sua madre e con lei è stato molto buono e generoso.

Il bambino comincia a esplorare i dintorni, si rende conto che il recinto è molto più lungo di quello che vede dalla sua finestra. Un giorno vede un punto che più da vicino diventa un bambino vestito con il pigiama a strisce. Si chiama Shmuel, ha nove anni, e presto fa amicizia con lui. Shmuel gli racconta che lui e gli altri non sono in questo posto per loro volontà, che si trovano in Polonia e che un giorno furono tutti ammucchiati in treno, separati da madri e da sorelle e portati lì. Ma non si sa perché. I grandi lavorano: oltre alle donne messe a fare le domestiche nella casa di Bruno, viene un uomo a pela- re le verdure e a servire a tavola, ma quando Bruno cade dalla sua altalena, lo cura e si rivela medico. C'è anche un personaggio molto negativo, un giovane tenente diciassettenne che tratta Bruno con disdegno. Un giorno quando $\mathrm{Pa}$ vel, il dottore cameriere, per debolezza si lascia cadere dalle mani la bottiglia di vino si arrabbia molto e lo offende.

Prima della nuova amicizia del padre con Fury, i genitori di Bruno davano feste sontuose con la partecipazione della nonna, una cantante famosa e del nonno, proprietario di un ristorante. Durante l'ultima festa con i nonni prima di partire per Out-With, la nonna aveva manifestato il suo disappunto per il lavoro e l'atteggiamento di suo figlio, diventato una marionetta nelle mani dei superiori. Anche la madre di Bruno non è più molto felice. Si intuisce inoltre dal suo rapporto con il giovane tenente che oltrepassa le regole di buona condotta della moglie di un comandante.

Quando Bruno e la sorella contraggono i pidocchi, la madre riesce a convincere il padre che forse quello non è il luogo adatto per tirare su i figli e decide così di tornare con loro a Berlino. La testa di Bruno viene rasata, e quando va a salutare Shmuel, le due teste si assomigliano. Sono nati lo stesso giorno e lo stesso anno, ma Bruno è un bambino forte e sano, anche se piccolo di statura, mentre Shmuel è denutrito al massimo.

L'ultimo giorno prima della partenza per Berlino decidono di giocare insieme agli esploratori: adesso Bruno, avendo la testa rapata, può passare per uno di loro, se si mette un pigiama a righe. Shmuel lo asseconda perché ha smarrito suo padre, così Bruno gli promette di aiutare a cercarlo. Alzano il recinto ed ecco Bruno in mezzo a 
tutta quella gente impaurita e triste. I ragazzi si ritrovano in un gruppo di adulti a cui è stato ordinato di avanzare. I due piccoli si fanno trascinare dai grandi e si ritrovano in una stanza piena di gente. Si tengono per mano quando si chiude la porta.

Un anno dopo il padre ha un'intuizione: nel punto dove sono stati trovati gli stivali e altri indumenti di Bruno vede che il recinto è stato sollevato e può seguire i passi compiuti da suo figlio.

\section{Un paragone}

Cosa hanno in comune i due romanzi? I padri sacrificano i loro figli nella ferrea logica di seguire un'idea. Ovviamente vogliono loro un gran bene, però non li ascoltano. Michele e Bruno fanno intendere che hanno trovato un amico nel mondo, che gli adulti giudicano avversario, gli portano cibo e acqua e gli danno affetto. Queste azioni dovrebbero ispirare i padri a lasciare il loro progetto distruttivo, ma questi sono talmente presi dal loro ruolo nel progetto da non percepire minimamente questi segnali. Il padre di Michele ha la fortuna che suo figlio non morendo lo salva dall'ergastolo; se il colpo di pistola fosse stato più preciso sarebbe morto. Il padre di Bruno, responsabile del campo di Auschwitz, non poteva evitare la finale tragica di suo figlio.

Non è una coincidenza che i due protagonisti abbiano nove anni. A quest'età il bambino interiorizza il cosiddetto super-ego, i valori e la morale predominanti nella società in cui vive. Ha una nitida visione di ciò che è giusto e ciò che è sbagliato, e normalmente nel trasferimento di queste idee il padre rappresenta un ruolo fondamentale.
Si presenta una domanda: perché il padre di Michele e il padre di Bruno sono coinvolti in azioni negative e omicide? Nella società patriarcale essi sono i responsabili del benessere della famiglia e le madri sono complici più o meno passive, sempre nel nome del progresso materiale e sociale. I bambini a nove anni già pensano in maniera indipendente e in più subiscono il fascino di infrangere un divieto. Michele e Bruno non capiscono perché non debbano frequentare i nuovi amici e i loro padri non danno una spiegazione valida e soddisfacente. La tragedia sta nel fatto che i due padri sono ben coscienti di essere coinvolti in azioni contrarie al modello che vorrebbero rappresentare per i figli. Il padre di Michele sta scivolando nel sequestro di un bambino, ma è sicuro che sia una cosa da niente e che con i soldi ricavati la famiglia avrebbe la possibilità di vivere più agiatamente. Alla domanda di suo figlio sulla ragione del sequestro dà una della risposte preferite dei genitori ai figli quando non sanno cosa dire: "tu sei troppo piccolo per capire". Il padre di Bruno parla di esseri non veramente umani, ma lui stesso non crede davvero al suo ruolo ingiustificabile. Sua madre gli aveva insegnato un altro stile di vita e altri valori.

\section{II figlicidio nella letteratura religiosa e mitologica}

Il tema del figlicidio fa parte della letteratura, come il parricidio, matricidio, fratricidio e infanticidio; la letteratura riflette la vita reale. Però il sacrificio del figlio da parte del padre è tanto endemico che, di contro, si può considerare quasi tabù. Uno dei primi 
personaggi che viene in mente è Abramo che non si nega agli ordini di Dio ed è pronto a sacrificare Isacco. E perché? Cosa sarebbe successo se avesse discusso con Dio sul senso di questo sacrificio? Non si sa, lui ha eseguito gli ordini fino a ricevere il contrordine.

Inoltre c'è Dio che ha sacrificato suo figlio sulla croce, un atto che ancora oggi risulta incomprensibile alla mente comune. Era davvero necessario sacrificare il figlio per salvare noi peccatori? $\mathrm{E}$ la salvezza non si basa sul pentimento e il successivo perdono? O si tratta del perdono del peccato non espiato? O sul perdono per il proprio bene, come una purificazione per stare in pace con se stessi? O ritorna l'eterna contrapposizione tra il bene e il male, come nel Vangelo di Giovanni, dove sta per vincere il male (la morte) e dopo vince il bene (la resurrezione)? Nel Vangelo di Luca Gesù non era cosciente di dover morire per redimere le anime peccatrici dell'umanità. Nella preghiera insegnataci e tramandataci, il Padre Nostro, con "rimetti a noi i nostri debiti, come noi li rimettiamo ai nostri debitori", rimane chiaro che il perdono avviene nel rapporto tra l'uomo, Dio e il suo prossimo. Durante l'ultima cena dice che il suo corpo e il suo sangue sono dati per gli apostoli, ma nel Vangelo di Luca non specifica che sarebbe per la salvezza dai peccati. E nel Vangelo di Giovanni non se ne parla affatto. Praticamente sapeva che doveva morire per le sue idee e per il suo messaggio, una morte non riservata solamente a lui nel mondo, ancora oggi pieno di ingiustizie.

Comunque doveva essere un gran dolore sapere di dover morire sul patibolo senza avere una colpa. L'interlocutore di Corrado Augias (1935-) nella sua indagine storica sulla fisionomia di
Gesù, Inchiesta su Gesù , è il biblista Mauro Pesce. Questi sostiene che Gesù era in primo luogo un ebreo praticante che si concentrava molto sulle preghiere e sollecitava Dio chiedendo risposte su cosa volesse da lui, attraverso visioni, rivelazioni e trasfigurazioni. Si distingueva per la sua assoluta fiducia in Dio, per la misericordia, per l'amore ai più emarginati e deboli, per la sete dell'armonia, della riconciliazione e della giustizia. Come taumaturgo poteva aiutare e guarire, ma non riusciva a comprendere perché Dio lo volesse ucciso in modo atroce. Accetta la sua missione e l'abbandono del padre sulla croce, anche se a occhi umani appare incomprensibile.

Si potrebbe eventualmente considerare la tesi di José Saramago (19222010) nel suo Il Vangelo secondo Gesù Cristo, dove Dio fa crocifiggere suo figlio per aumentare il suo potere e la sua influenza su un popolo piccolo come gli ebrei fino a diventare il Dio universale di tutta l'umanità. Altrettanto interessante è l'ipotesi che Gesù si sia sacrificato per la colpa che sentiva per la morte di molti bambini maschi, dopo che Erode lo aveva ordinato. Il padre Giuseppe, per redimersi nell'aver saputo di questo piano e non aver avvisato gli altri genitori, si unisce a un gruppo di combattenti contro il regime romano e viene crocifisso.

Un'altra interpretazione potrebbe anche essere che Dio non ha sacrificato suo figlio, visto che se lo è ripreso e adesso sta seduto accanto a lui, come dice il Credo. Poi si potrebbe dire che Dio si è identificato con gli uomini attraverso suo figlio e vive tutti gli aspetti della vita umana, morte compresa; dopodiché il figlio risuscita. Questo potrebbe significare la rinascita o immortalità dell'uomo stesso. 
Secondo gli indú, Dio (Brahman) si manifesta in tre parti (Trimurti): la creativa, Brahma, la conservativa, Visnu, e la distruttiva, Siva. Questo rispecchia forse più precisamente la natura umana. E allora si spiega il padre come creatore-sostenitore-distruttore.

Nella mitologia greca Crono mangia i suoi figli per paura di essere mangiato da uno di loro, e si salva solamente Zeus che si nasconde. Tantalo figlio di Zeus e il preferito degli dei, uccide suo figlio Pelope e lo presenta in pasto agli dei invitati. D'altro canto Laio abbandona suo figlio neonato con i piedi legati perché muoia sulle montagne: vuole evitare l'oracolo che prediceva la sua morte per mano di suo figlio. Un pastore ebbe pietà della piccola creatura indifesa $\mathrm{e}$ la portò alla corte di un altro re dove crebbe con il nome di Edipo. Comunque, divenuto grande, Edipo arriva alla corte del padre Laio e lo uccide per unirsi con sua madre. Questo è un tema che si ripete nelle varie forme letterarie ed è fonte di tanta letteratura già dal periodo dei greci. L'anello di congiunzione si puó trovare nel medioevo con l'Hildebrandslied, che tratta dell'incontro di un cavaliere con il figlio abbandonato nell'infanzia. Il figlio crede che il padre sia un eroe morto in guerra e non ammette la sua paternità. La provocazione del figlio induce il padre, intaccato nell'onore, al duello e il figlio muore ucciso.

\section{Il figlicidio nella narrativa e nella drammaturgia}

Proseguendo avanti nel tempo, nel secolo d'oro della letteratura spagnola, nel '600, Pedro Calderón de la Barca (1600-1681) scrisse una commedia, La vida es sueño, dove gli astrologi progno- sticano al re di Polonia che suo figlio lo avrebbe ucciso. Perciò, questi, come il padre di Edipo, ripudia suo figlio e lo fa crescere lontano dalla corte. Già vecchio, rendendosi conto che i nipoti non sono adatti a prendere il suo ruolo, vuole mettere a prova il figlio e lo fa riportare dalla cella al palazzo. Quando si sveglia tutti gli rendono omaggio ma lui si manifesta aggressivo e violento a corte. Non riesce a uccidere il padre che lo fa rinchiudere di nuovo, drogato. $\mathrm{Al}$ risveglio si rende conto che il male non porta bene e si pente di tutto quello che ha fatto nel suo sogno da figlio di re. $\mathrm{E}$ così questa storia è a lieto fine; il re e il figlio si abbracciano.

Nell'Ottocento i fratelli Grimm (Jakob 1785-1863 e Wilhelm 1786-1859) raccolsero le favole del loro mondo, dove tanti bambini, a cominciare da Hansel e Gretel, si ritrovavano soli e abbandonati nel bosco; il loro padre sembra compiere quest'atto per la cruda mancanza di cibo. In altre favole come Le tre piume o I tre linguaggi il figlio doveva dimostrare le sue capacità a un padre modello, autoritario, depositario delle norme, giudice e maestro, oppure doveva affrontare la sua vita da solo e superarlo.

Uno degli esponenti letterari tedeschi più illustri del Settecento e dell'Ottocento, Friedrich Schiller (1759-1805), scrisse due tragedie che trattano della morte del figlio davanti al padre. I ladri parla di due figli, Karl e Franz. Karl è un fuorilegge che chiede perdono al padre, ma Franz interferisce per gelosia e non gli fa pervenire una sua lettera. Quando il padre piange per il figlio perduto, egli cerca di convincerlo dello spreco di sentimenti su di un figlio traditore di tutti i valori civili. Karl crede che il padre non 
lo voglia perdonare e giura lealtà alla sua banda di ladri. Quando alla fine lo incontra e si rende conto dell'errore, è già troppo tardi. Si consegna alla giustizia, dove sconta i sui delitti con la morte. Franz si toglie la vita quando si rende conto di aver perduto la sua causa e alla fine il padre rimane solo.

In Don Carlos, Infante di Spagna, il re Filippo II si lascia influenzare dai suoi consiglieri e cortigiani che interpretano l'invaghimento del figlio Carlos con la regina Elisabetta come un complotto verso di lui. Lei è stata sua amica d'infanzia e promessa sposa, prima che la sposasse il re stesso. Il padre vecchio e despota non ha mai avuto un buon rapporto con suo figlio e si ingelosisce: gli nega di andare nei Paesi Bassi per domare la ribellione e di contrasto Carlos è pronto a recarsi in quelle terre lontane per partecipare alla sommossa dal lato dei ribelli. Le tonache lo accusano di tradimento e il re condanna a morte il figlio.

In entrambi i drammi la comunicazione tra padre e figlio o non esiste affatto o è molto ristretta, in modo che il figlio non può chiarirsi o giustificarsi. I padri si lasciano influenzare da persone con scopi che contrastano con gli interessi dei figli. Di nuovo, il padre non vuole eliminare il figlio, ma la sua indisponibilità al dialogo lo porta a questa fine.

Paragonando Schiller con l'altro e più universalmente conosciuto scrittore tedesco suo contemporaneo, Johann Wolfgang von Goethe (1739-1832), emerge che quest'ultimo non tratta mai del conflitto tra padre e figlio. La vita di Goethe era complessivamente una vita felice, nato e cresciuto nel seno di una famiglia borghese molto benestante, con un padre esclusivamente dedicato all'educazione dei figli. Non ha dovuto lottare per avere una vita agiata ed è morto alla venerabile età di ottantadue anni. Schiller invece aveva un padre medico dell'esercito che costringeva suo figlio a studiare medicina per seguire le sue orme. Schiller è dovuto fuggire per potersi dedicare alla produzione letteraria e ha così sofferto per quasi tutta la vita di problemi di salute, morendo a quarantacinque anni. Questo fatto sta a dimostrare come ogni scrittore di narrativa includa nella sua opera una parte della propria esperienza.

Facendo un salto nell'epoca esistenzialista di circa un secolo fa, non si può ignorare il conflitto tra padre e figlio nelle opere di Franz Kafka (1883-1924). L'autore stesso aveva un rapporto difficile con il padre, come emerge dalla sua Lettera al padre mai spedita o consegnata. Il padre gli faceva sentire la sua inferiorità e la sua colpa per non corrispondere alle aspettative poste in lui e allora il figlio accusava il padre di averlo intimorito e aver pregiudicato la sua autostima. Il senso di colpa e di vergogna verso il padre si è manifestato successivamente anche nei rapporti con gli altri. Il padre è il tiranno che si è fatto da sé, commerciante di successo, pieno di energia e convinto del suo valore e della sua verità. Non tollera alcuna forma di opposizione. La tragedia consiste nel fatto che il padre non si rende conto della sua insensibilità e soffre della lontananza dei figli, credendo di avere la coscienza più che pulita per aver provveduto al sostegno adeguato della famiglia. Kafka riconosce che il padre non ha colpa, ma nemmeno lui si sente colpevole per la mancanza d'amore nei confronti del padre.

Molto drammatica diventa la situazione ne La Condanna, dove il padre 
debole e dipendente si trasforma in un uomo forte e despota: condanna suo figlio a morire annegato nel fiume che passa davanti alla casa. Nel surrealismo kafkiano il pretesto è quello di non aver comunicato all'amico in Russia il suo sposalizio prossimo. Ne La Metamorfosi, il padre passa da vittima reduce e debole, che lascia al figlio la responsabilità di sfamare la famiglia, a padre attivo e dominante, quando il figlio si riduce in mosca-scarafaggio - un insetto non meglio identificato e inutile. Il padre si rifiuta di riconoscerlo e influenza moglie e figlia affinché anche loro lo abbandonino.

Per quanto riguarda la letteratura italiana classico-moderna, due libri parlano del rapporto conflittuale tra padre e figlio. Padre padrone di Gavino Ledda (1938-) fa presente la durezza schiacciante di un'infanzia da pastorello, nell'entroterra sardo. Il padre toglie il figlio non ancora di sei anni dalla scuola per metterlo a pascolare le pecore, in una natura tutt'altro che incantevole. Il padre possiede già un oliveto, una vigna e altre coltivazioni da lavorare e ha anche la moglie e gli altri figli più piccoli da sfamare. Il bambino deve imparare la vita del pastore in fretta nonostante i suoi vari e inutili tentativi di fuggire. Nel suo primo inverno in una capanna esposta alle intemperie meteorologiche più estreme, contrae una doppia broncopolmonite e si salva più per forza vitale propria che per cure mediche. Un giorno quando il padre va in paese, il bambino, invece di pulire l'ovile dal letame, va a sentire il pastore vicino che canta. Al ritorno il padre quasi lo ammazza di botte in senso letterario; sta per perdere la facoltà visiva e di nuovo si salva più per volontà sua che per interventi esterni. Il padre pro- segue sfruttando e abusando di tutta la famiglia nella casa di campagna. Alla fine risulta che non era necessario che tutti i figli rinunciassero alla scuola e alla vita in paese, dato che il padre non solo non stava economicamente male, ma stava anche accumulando un bel gruzzolo come usuraio. In questo libro i figli alla fine non soccombono, si ribellano e si liberano di lui ma hanno sacrificato una parte importante della loro vita e subito dei traumi molto nocivi. L'unica attenuante per il padre consiste nel fatto che è cresciuto così anche lui, ricalcando gli schemi familiari. In questo caso si presenta la tendenza nella vita a seguire schemi e modelli imparati nell'infanzia e la difficoltà di superarli con la piena coscienza di agire per il bene proprio e altrui. Il conflitto e confronto tra padre e figlio è importante nella letteratura e nella vita; se il figlio non muore o soccombe, avanza e progredisce. Viene in mente un altro classico, La coscienza di Zeno di Italo Svevo (1861-1928), dove il padre è cosi potente ed egoista che il figlio trascorre i suoi giorni facendo finta di studiare e fumando in continuazione. Il padre disprezza il figlio e in punto di morte gli dà uno schiaffo come segno della sua incomprensione. Dopo il lutto, il figlio diventa emotivamente e economicamente dipendente dal ragioniere e dal suocero; non riesce inoltre a sviluppare una autostima e soffre di malattie reali e immaginarie. Alla morte di questi due personaggi riesce poco a poco a prendere lui il posto di pater familias e a redimersi.

Interessante risulta il fatto che il protagonista si sottopone a una psicoanalisi in cui l'analista gli scopre il complesso di Edipo, seguendo la teoria di Sigmund Freud (1856-1939), padre 
della psicologia moderna e contemporaneo di Svevo. Secondo lui quasi tutti i problemi esistenziali dell'uomo sono causati da impulsi sessuali repressi e nel caso particolare dei maschi dal desiderio di unirsi alla madre. Zeno, pur avendo bellissimi ricordi di questa, non accetta questa teoria e comincia infatti a riprendersi quando si libera anche dall'analista, pur non essendo stato dichiarato guarito.

Questa teoria di Freud ha comunque perso molto del suo valore, accreditato durante la società vittoriana, sessualmente molto repressa. È vero che molto spesso il figlio da piccolo vive il suo primo grande amore per la madre e può anche dire che da grande vuole sposarla, ma questo non significa per forza che voglia eliminare il padre. Nella fantasia infantile possono vivere insieme tutti e tre per sempre.

Per passare al prossimo argomento dei casi di sacrifici nella vita, ecco un autore e filosofo famoso, Jean-Jacques Rousseau (1712-1778), che ha elaborato un sistema educativo rivoluzionario nell'Emile. Sosteneva che l'uomo per natura è buono e che la società lo rende cattivo; perciò insisteva nell'educazione libera nella natura e sul rispetto degli interessi e delle inclinazioni del bambino. Purtroppo nella vita questo grande pensatore non si è mostrato molto premuroso verso i suoi figli, ma li ha lasciati tutti e cinque all'orfanotrofio. Non si è più saputo niente di loro. Normalmente in quei tempi non molti bambini dell'orfanotrofio arrivavano vivi all'età adulta.

\section{Rapporti padre-figlio nella vita}

E' necessario fare qui una premessa: fino a relativamente poco tempo fa, i figli non erano considerati sempre come individui con valori propri. I figli arrivavano e la loro vita acquistava valore solo da grandi; i figli erano sostenuti dal rapporto coi genitori nell' infanzia, cosicché da grandi dovevano sostenere loro i genitori. D’altronde la vita media raramente superava i quarant'anni, perciò era normale per un padre ripudiare un figlio che non gli conveniva; già presso gli spartani un infante con problemi seri veniva lasciato esposto in campagna o nel bosco, perfino gettato in una fossa per farlo morire. Si sa anche che spesso i padri vendevano i figli come schiavi.

Un altro personaggio della storia recente con la vita di un figlio sulla coscienza è Benito Mussolini (18831945). Già con Ida Dalser aveva avuto il figlio Benito Albino Dalser, quando come Duce aveva sposato Rachele e avuto figli legittimi con lei. Quando Benito Albino ha insistito sulla paternità lo ha fatto rinchiudere in un manicomio, dove è morto presumibilmente di consunzione nel 1942.

Passando all'altra sponda dell'Atlantico, gli aztechi e tutte le culture mesoamericane sacrificavano esseri umani: prigionieri di guerra, bambini, malati. Secondo la credenza azteca, gli dei si erano autosacrificati e per garantire la continuità della vita richiedevano dei sacrifici umani, apparentemente uno per ognuno dei loro diciotto mesi dell'anno.

Inoltre ci sono tanti padri che mandano i figli in guerra, nel nome di cosa? Ciò non corrisponde ai comandamenti e neanche all'etica cristiana della famiglia -al momento del matrimonio gli sposi vengono incoraggiati a moltiplicarsi.

Il sacrificio in sé è ammirevole e nobile, sempre quando la persona decide 
di sacrificarsi per aiutare o salvare gli altri, come chi per salvare un bambino dal fuoco muore bruciato. Ma nessuno ha il diritto di sacrificare la vita degli altri, nemmeno dei propri figli. Ovviamente ci sono anche casi dove il padre si sacrifica per salvare il figlio, di nuovo è lui che prende la decisione su cosa fare della sua vita.

$\mathrm{Ci}$ sono modi più perfidi per distruggere un figlio: il padre lo educa e lo prepara a succedergli negli affari, anche se il figlio ha altre doti e interessi. Questo per fortuna ai giorni d'oggi nel mondo occidentale è meno frequente; negozi privati e botteghe artigianali falliscono costantemente in mancanza di un successore.

Una considerazione speciale spetta ai figli di uomini cosiddetti geniali, straordinari. Normalmente non hanno le stesse doti dei loro padri e non riescono a uscire dall'ombra paterna. È una legge non scritta che il figlio, se non supera il padre, rimanga almeno allo stesso livello per quanto riguarda il successo professionale (esteriore) e quello personale (interiore). Sempre lo scrittore Thomas Mann aveva sei figli, Klaus si tolse la vita a 43 anni e Michael a 58 anni. D'altro canto i padri di bambini prodigio come Leopoldo Mozart, padre di Wolfgang Amadeus, sono portati a sfruttare i talenti dei figli.

Non è mia intenzione in questo luogo di brutalizzare la figura del padre; come già detto sopra, fino ai tempi recenti l'unità familiare era molta salda e uno o più figli imparavano il mestiere dal padre per poi seguire le sue orme; nessuno metteva in discussione se questo corrispondeva agli interessi del figlio, nemmeno il figlio stesso. E normalmente questo ordine non era traumatico ma accettato come destino. Tutt'oggi ci sono ancora molti casi in cui il figlio sceglie di seguire il padre, con successo e soddisfazione. Soprattutto se il padre dimostra di essere felice e realizzato nel suo ruolo nel mercato imprenditoriale, diventa così una motivazione per il figlio l'imitarlo. Inoltre, attualmente il padre prende il ruolo di consigliere del figlio, lo appoggia e lo incoraggia, a differenza dei modelli molto rigidi e spartani dell'educazione fino a un secolo fa. Il padre dimostra il suo affetto apertamente, con attenzione e coccole e nel migliore dei casi contribuisce a creare un figlio realizzato e felice. Comunque, considerando lo stato imperfetto evolutivo di ogni essere umano, anche i genitori con le migliori intenzioni commettono errori, provocando traumi seri nei figli compromettendo il loro futuro.

Infine, come per tutti i crimini, esistono vari modi di eseguirli: involontariamente o accidentalmente oppure per negligenza, come nei due romanzi considerati, o appositamente. I padri di Michele e Bruno non avevano nessuna intenzione di uccidere i figli, e questo può considerarsi un'attenuante, ma rimangono comunque colpevoli .

Un'ultima considerazione va fatta riguardo alle madri: è ben saputo che anche loro possono essere capaci di sacrificare i figli, ma questo sarà un tema per un'altra occasione.

\section{Bibliografia}

Ammaniti, N. (2001). Io non ho paura. Torino: Einaudi Stile Libero, Giulio Einaudi editore.

Augias, C. (2006). Inchiesta su Gesù. Milano: Arnoldo Mondadori Editore. Augias, C. (2013). Inchiesta su Maria. Milano: RCS Libri. 
Boyne, J. (2012). The Boy in the Striped Pyjamas. Great Britain: Random House Children's Publishing.

Kafka, F. (2011). Franz Kafka: Sämtliche Werke, Band I. Deutscher Literaturhaus-Verlag.

Lagarde e Michard (1970). XVIIIe Siècle: Les grands auteurs français du programme IV. Parigi-Montreal: Bordas.

Saramago, J. (2006). El Evangelio según Jesucristo. México: Punto de Lectura.
Schiller, F. Die Räuber. Urheberrechtsfreie Ausgabe.

Schiller, F. Don Carlos, Infant von Spanien. Urheberrechtsfreie Ausgabe.

Schwab, G. Sagen des klassischen Altertums. Urheberrechtsfreie Ausgabe.

Svevo, I. (1918-1923). La coscienza di Zeno. Guidaebook.com, servizio di editing digitale. 